Artículo

\title{
Rendimiento y calidad de fruto de fresa cultivada en invernadero en sistema hidropónico piramidal
}

Juan Alberto Alvarado-Chávez

Adrián Gómez-González ${ }^{1}$

Alfredo Lara-Herrera ${ }^{2}$

J. Carlos Díaz-Pérez ${ }^{3}$

E. Javier García-Herrera ${ }^{1 \S}$

${ }^{1}$ Innovación en Manejo de Recursos Naturales-Campus San Luis Potosí-Colegio de Postgraduados. Calle Iturbide núm. 73. CP. 78600. Salinas de Hidalgo, San Luis Potosí, México. (albertokromo@ hotmail.com; agomez@colpos.mx). ${ }^{2}$ Universidad Autónoma de Zacatecas. Jardín Juárez \#149, Centro Histórico, Zacatecas, Zacatecas, México. CP. 98000. (alara204@hotmail.com). ${ }^{3}$ Departamento de HorticulturaUniversidad de Georgia. 2360 Rainwater Road, Tifton, Georgia, Estados Unidos de América. 31793-5766. (jcdiaz@uga.edu).

${ }^{\S}$ Autor para correspondencia: garciae@colpos.mx.

\section{Resumen}

La fresa (Fragaria x ananassa Duchesne) es un cultivo importante en México y el mundo. Para incrementar el rendimiento se han explorado varios sistemas de producción, como los sistemas hidropónicos. El objetivo de este estudio fue determinar el efecto de cultivares de fresa, densidades y estratos de plantación en sistema hidropónico piramidal, en el desarrollo de las plantas, producción y calidad de frutos. El estudio se realizó en Salinas de Hidalgo, SLP, México, en el ciclo primavera-otoño de 2017. El factor estrato impacto en el desarrollo y rendimiento del cultivo, con el estrato alto se incrementó el número de hojas, ancho de la corona, número de frutos y rendimiento $\left(23.5 \mathrm{~kg} \mathrm{~m}^{-2}\right.$ ), y cantidad de sólidos solubles en comparación con los inferiores. Las plantas en estratos intermedios fueron menos productivas, probablemente por la sombra causada por las de estrato más alto. La densidad y el cultivar no tuvieron efecto significativo en el desarrollo, rendimiento y calidad de fruto.

Palabras clave: Fragaria x ananassa Duchesne, densidad de plantas, invernadero, sistemas verticales de producción.

Recibido: octubre de 2020

Aceptado: diciembre de 2020 


\section{Introducción}

Existe creciente interés en la inclusión de frutillas, especialmente fresa en la dieta diaria de las personas, principalmente por los beneficios en la salud asociados con su consumo (Çolak y Alan, 2017). En 2008, a nivel mundial, la fresa fue cultivada en 322630 ha con una producción de 5978807 t, en 2018, se cultivó en 372361 ha, con una producción anual de 8337099 t. Actualmente, México es el tercer país productor, después de China y Estados Unidos de América, con 7.8\% de la producción mundial. En 2018, se plantaron en México 13652 ha con una producción de 653639 t (FAOSTAT, 2020).

Las técnicas de producción intensivas como los sistemas hidropónicos han incrementado su uso (Hernanz et al., 2008). Uno de los cultivares más promovidos en México es 'Festival'; este cultivar es de fotoperiodo corto, fruta en forma cónica y de color rojo intenso (Chandler et al., 2000). El cultivar 'San Andreas' de reciente introducción, es de fotoperiodo moderadamente neutro, con poca necesidad de frío en vivero (Eurosemillas, 2020).

López-Pérez et al. (2005) mencionan que en los 1990's, se exploraron varias formas de incrementar el rendimiento en el cultivo de fresa en condiciones tradicionales en suelo. Actualmente se emplean los sistemas hidropónicos, por sus beneficios con la utilización más eficiente de fertilizantes y plaguicidas (Keutgen y Pawelzik, 2007), altas densidades, adecuado balance de nutrientes (Sánchez del Castillo et al., 2014) y agua (Van Ginkel et al., 2017), mayor rendimiento (Paranjpe et al., 2008; Tariq et al., 2013) y calidad de fruto (Caruso et al., 2011).

En estos sistemas se puede recurrir al agua como medio de cultivo o diferentes sustratos, tales como lana de roca, fibra de coco o perlita; La fertilización es mediante soluciones nutritivas (Kratky, 2005). La densidad de fresa en suelo es de 6.5 a 8 plantas $\mathrm{m}^{-2}$, mientras que en los sistemas hidropónicos verticales puede ser hasta tres veces mayor (Ozeker et al., 1999). Como los bastidores metálicos y arreglos en bolsas verticales, pudiendo producir la mayoría de los cultivos hortícolas, tales como lechuga, tomates y fresas (Benke y Tomkins, 2017).

La producción de fresa puede aumentar con el incremento de densidad de plantas. Paranjpe et al. (2003) obtuvieron rendimientos de $9 \mathrm{~kg} \mathrm{~m}^{-2}$ en invernadero sin control de clima con densidad de 20 plantas $\mathrm{m}^{-2}$ cultivadas en bolsas con sustrato en Florida, EUA. Hay estudios acerca de calidad de fruto en fresa cultivada en sistemas hidropónicos (Cecatto et al., 2013; Karimi et al., 2013), así como la influencia del sustrato en la producción y calidad de frutos (Recamales et al., 2007; Palencia et al., 2016; Martínez et al., 2017). El objetivo de este estudio fue determinar el efecto de cultivares de fresa, densidades de plantación y estratos de plantación en sistema hidropónico piramidal, en el desarrollo de plantas, producción y calidad de frutos.

\section{Materiales y métodos}

El estudio se realizó en un invernadero bitúnel, con ventanas laterales manuales, altura de $5 \mathrm{~m}$ a la cúpula, y superficie de $756 \mathrm{~m}^{2}$, sin control climático. La temperatura del aire y humedad relativa del invernadero se midieron con sensores conectados a un datalogger (Hobo, modelo U23 V2). El invernadero está ubicado en el municipio de Salinas de Hidalgo, San Luis Potosí, México [22 $37^{\prime}$ 39' latitud norte, $101^{\circ} 42$ ' 52' longitud oeste; altitud de 2070 m (INEGI, 2020)]. 
Los cultivares utilizados fueron 'Festival' y 'San Andreas'. La planta a raíz desnuda se adquirió con productores del municipio de Pabellón de Arteaga, Aguascalientes, México. Se mantuvo en refrigeración a $3{ }^{\circ} \mathrm{C}$ durante 60 días antes del trasplante para su almacenamiento. Se plantó el día 10 de marzo de 2017 de manera directa. Para establecer el experimento se comenzó con la construcción del sistema hidropónico y la colocación de las bolsas horizontales con fibra de coco.

El sistema piramidal se fabricó con una estructura de acero, con tres tubos de metal de $1.5 \mathrm{~m}$ de largo cada uno, los triángulos tienen ocho tubos cortos de $0.22 \mathrm{~m}$, soldados a los lados a cuatro alturas cada $0.5 \mathrm{~m}$, los cuales sirven para colocar dos tramos de tubo de $1 \mathrm{~m}$ de largo, que funcionan como base para colocar las bolsas (Figura 1). Cada repetición se conformó por tres módulos. Las bolsas con fibra de coco horizontales son Germinaza ${ }^{\circledR}$ con la mezcla de $50 \%$ polvo y $50 \%$ fibra, de $1 \mathrm{~m}$ de largo por $0.2 \mathrm{~m}$ de ancho con un volumen de $18 \mathrm{~L}$. Se utilizaron 24 bolsas horizontales por repetición.

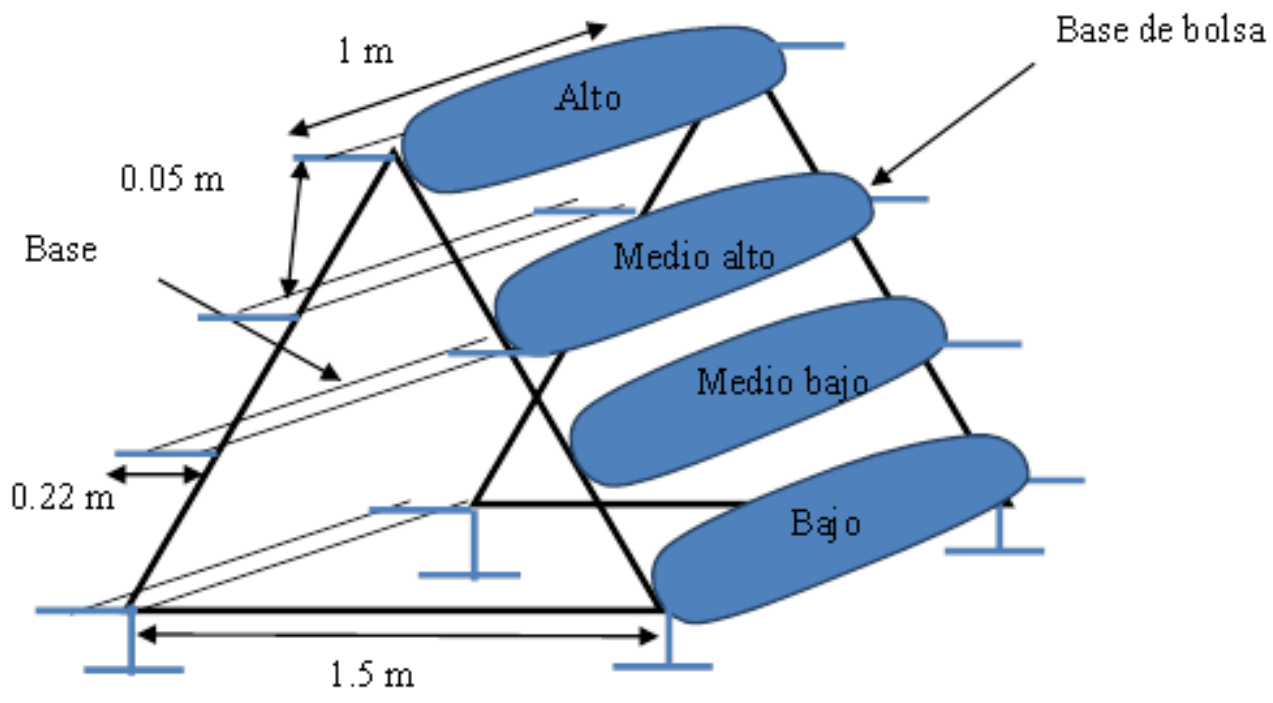

Figura 1. Esquema del módulo hidropónico piramidal, con la estructura y las bolsas horizontales ubicadas en cuatro estratos.

El sistema de riego consistió en cintilla de flujo de $0.5 \mathrm{~L} \mathrm{~h}^{-1}$ y una distancia entre goteros de $10 \mathrm{~cm}$, conectada a una bomba de $1 / 4$ de hp por medio de manguera de $16 \mathrm{~mm}$. El sistema se automatizó para dar 2 o 3 riegos por día según lo requirió el cultivo. El sistema de fertirriego estuvo abastecido de solución nutritiva por un reservorio de 2500 L.

En cuanto al manejo de la solución nutritiva, durante los primeros tres días se regó sólo con agua de lluvia, posteriormente se regó con solución nutritiva. Se colocaron las bandejas para recabar el drenaje y los goteros de control en cada estrato, las mediciones del volumen, $\mathrm{pH}$ y conductividad eléctrica se llevaron a cabo dos veces por semana del 15 abril al 6 de octubre de 2017.

Se utilizó la solución nutritiva de Hewitt y Smith (1974); Caruso et al. (2011). Mediante los fertilizantes: nitrato de potasio $\left(\mathrm{KNO}_{3}\right)$, nitrato de calcio $\left(\mathrm{Ca}\left(\mathrm{NO}_{3}\right)_{2}\right.$, fosfato monopotásico $\left(\mathrm{KH}_{2} \mathrm{PO}_{4}\right)$, sulfato de magnesio heptahidratado $\left(\mathrm{MgSO}_{4} 6 \mathrm{H}_{2} \mathrm{O}\right)$, sulfato de potasio $\left(\mathrm{K}_{2} \mathrm{SO}_{4}\right)$, como fuente de micronutrientes se utilizó una mezcla comercial de nombre Tradecorp ${ }^{\circledR} \mathrm{AZ}$. El pH de la 
solución nutritiva se mantuvo en $6 \pm 0.3$, y conductividad eléctrica de $1.3 \mathrm{dS} \mathrm{m}^{-1}$ en etapa vegetativa ( 1 a 20 semanas después del trasplante, SDT) y $1.5 \mathrm{dS} \mathrm{m}^{-1}$ en la etapa reproductiva (21 a 30 SDT) aplicada de 100-400 ml planta ${ }^{-1}$. El suministro de nutrientes se manejó con un sistema abierto.

Para el control de plagas, cada dos semanas se realizaron podas de saneamiento, en la cual se retiraron hojas viejas y con daños por plaga. De mayo a agosto se controló el insecto Tetranychus urticae, mediante abamectina alternada con cipermetrina en dosis de $1 \mathrm{ml} \mathrm{L}^{-1}$. Las variables de desarrollo medidas fueron: número de hojas y diámetro de corona. El diámetro se midió de la parte ancha y se consideró a las múltiples coronas de la planta como una sola (Cantliffe et al., 2007). Las variables se midieron cada dos semanas a partir del día 5 de mayo, al 6 de octubre de 2017.

Las variables calidad y producción de fruto fueron determinados en cada cosecha mediante la medición de: diámetro con vernier digital y el contenido de sólidos solubles del fruto con un refractrómetro óptico Atago Brix 0.0 a 33\%) a partir del jugo del fruto, según la norma NMX-FF062-SCFI-2002, peso de fruto y rendimiento de fruto (por planta y metro cuadrado). La cosecha de frutos se realizó cada semana a partir del día 28 de julio y terminó el día 6 de octubre de 2017.

El diseño experimental fue en bloques completos al azar con 24 tratamientos y tres repeticiones con arreglo factorial. Los factores ensayados fueron: (a) dos cultivares de fresa ('Festival' y 'San Andreas'), (b) tres densidades de plantas por bolsa horizontal (6, 8 y 10, correspondientes a 33.2, 25 y $20 \mathrm{~cm}$ lineales entre planta con doble hilera de planta en tresbolillo y equivalentes a 32, 43 y 53 plantas $\mathrm{m}^{-2}$, respectivamente) y (c) cuatro estratos en el sistema piramidal (alto, medio alto, medio bajo y bajo). Se tomaron datos de dos plantas por tratamiento experimental con un total de 72 datos de cada variable.

El análisis estadístico de los resultados se realizó mediante un modelo general lineal, un procedimiento de regresión por medio del programa estadístico SAS versión 9.4. Con prueba de medias de Tukey al $95 \%$ de confianza.

\section{Resultados y discusión}

\section{Factores cultivar y densidad de plantas}

No se presentó efecto significativo en ninguna de las variables evaluadas para los factores: cultivares y densidad de plantas, tampoco para ninguna de las interacciones; solo hubo efecto significativo para el factor estrato de las plantas en el sistema hidropónico. Por lo cual, los resultados que se presentan corresponden a este factor.

\section{Factor estrato}

Variables de desarrollo. entre las 8 y 19 SDT las plantas mostraron comportamiento vegetativo, sin diferencia entre tratamientos en los cuatro estratos. Después de las 20 SDT las diferencias comenzaron para: número de hojas (Figura 2), diámetro de la corona (Cuadro 1), diámetro de fruto (Cuadro 2) y número de frutos (Cuadro 3). 
Las plantas del estrato alto tuvieron mayor número de hojas (Figura 2A). De acuerdo con el análisis de regresión, después de la semana 30, el número de hojas incrementó en el estrato alto, contrario a lo que se observó en los estratos más bajos (Figure 2B).

$2 \mathrm{~A}$

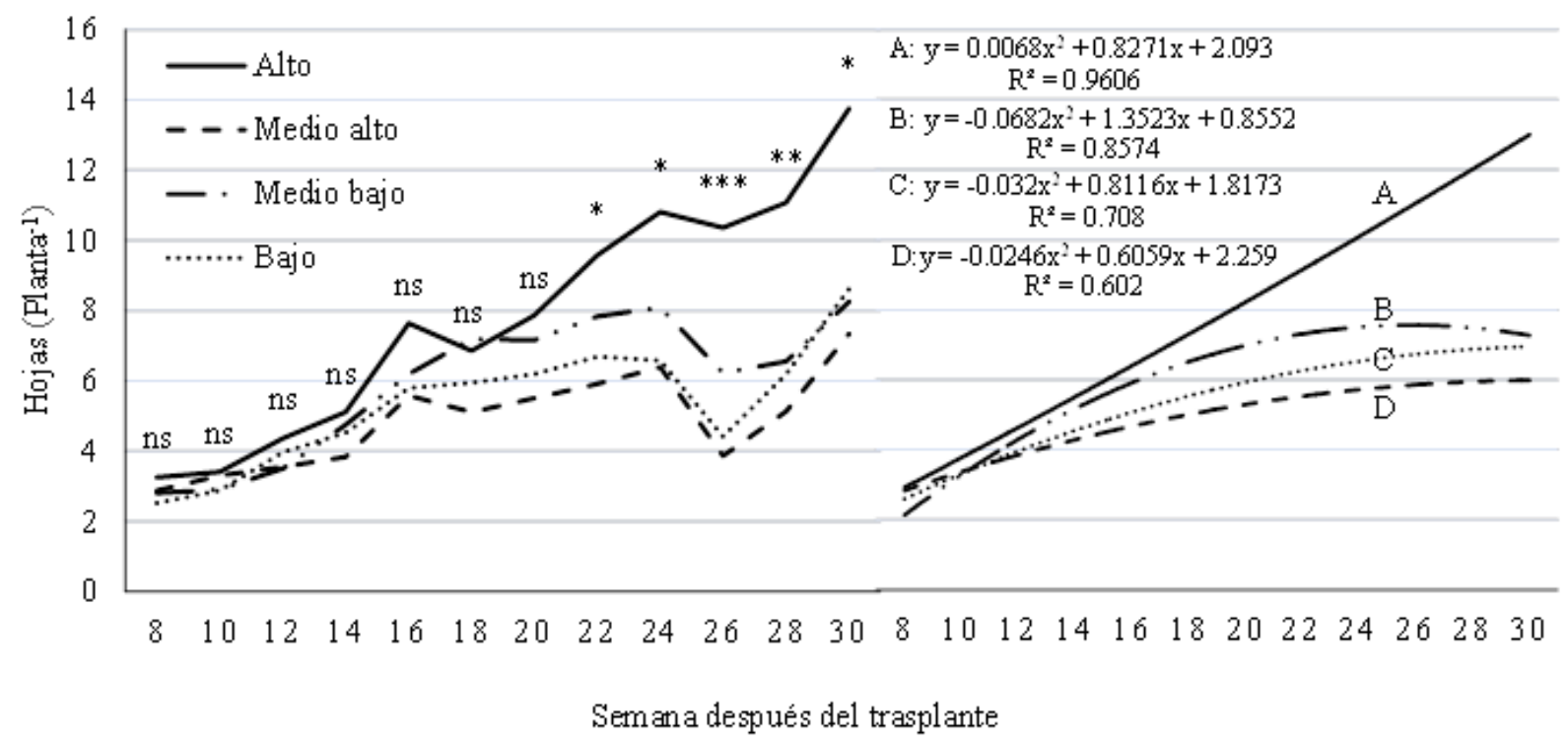

Figura 2. Número de hojas por planta de fresa de invernadero producida en cuatro diferentes estratos en sistema de producción con estructura piramidal. 2A) las curvas conectan las medias, datos evaluados mediante análisis de varianza (General Linear Model, SAS); 2B) curvas calculadas mediante regresión lineal. $\mathrm{ns},{ }^{*},{ }^{* *},{ }^{* * *}$ no significativo y significativo a $p \leq 0.05,0.01$ y 0.001 , respectivamente.

En el estrato alto, el número de hojas fue de 13 hojas a las 30 SDT; este valor es menor a las 16 hojas por planta reportado por Juárez-Rosete et al. (2007) en un sistema NFT horizontal a las 10 SDT, donde menciona que el NFT al ser un sistema circulante (cerrado) se le da un suministro constante de nutrientes a diferencia de los sistemas en sustrato abierto. Aunado a esto, Díaz-Pérez (2013) menciona que bajo sombra puede reducirse la productividad de los cultivos.

Esto concuerda con nuestros resultados en donde se observó un menor número de hojas y una reducción en el diámetro de la corona en los estratos más sombreados. La inclinación del sistema piramidal pudo ocasionar que las plantas se sombrearan en mayor medida en los estratos intermedios. El menor tamaño, de los estratos intermedios ocasiona que las plantas del estrato bajo tuvieron mayor exposición a la luz, ya que la inclinación evita que las plantas con menor crecimiento, en este caso las plantas de los estratos intermedios, afecten el desarrollo de las plantas en el estrato bajo.

A las 26 SDT se observó disminución del número de hojas, esto por la poda de saneamiento que se dió para estimular la formación de nuevo follaje y flores, con ello evitar formación de estolones, ya que es una de las causas por las que la planta de fresa pierde nutrimentos y compuestos fotosintetizados (Avitia-García et al., 2014). 
El análisis de regresión mostró incremento del número de hojas en el estrato alto en comparación con los tres estratos inferiores, es el único que muestra un comportamiento creciente, con una $\mathrm{R}^{2}=$ 0.9606. Los resultados del diámetro de la corona concuerdan con el comportamiento del número de hojas, en la mayoría de los casos, el mayor diámetro se presentó en el estrato alto de las plantas con $77 \%$ de incremento respecto a los estratos intermedios, donde fue menor (Cuadro 1).

Cuadro 1. Diámetro de la corona de fresa para dos cultivares en cuatro estratos en sistema hidropónico de producción piramidal, plantadas en bolsas horizontales.

\begin{tabular}{ccccccc}
\hline \multirow{2}{*}{ Factor estrato } & \multicolumn{6}{c}{ Semanas después del trasplante } \\
\cline { 2 - 7 } & 20 & 22 & 24 & 26 & 28 & 30 \\
\hline Alto & $2.43 \mathrm{a}^{\dagger}$ & $2.61 \mathrm{ab}$ & $2.66 \mathrm{a}$ & $2.63 \mathrm{a}$ & $2.77 \mathrm{a}$ & $3.09 \mathrm{a}$ \\
Medio alto & $1.81 \mathrm{bc}$ & $1.96 \mathrm{ab}$ & $2.03 \mathrm{ab}$ & $2.06 \mathrm{ab}$ & $2.04 \mathrm{ab}$ & $2.15 \mathrm{bc}$ \\
Medio bajo & $1.48 \mathrm{c}$ & $1.53 \mathrm{~b}$ & $1.69 \mathrm{~b}$ & $1.5 \mathrm{~b}$ & $1.54 \mathrm{~b}$ & $1.46 \mathrm{c}$ \\
Bajo & $2.1 \mathrm{ab}$ & $2.77 \mathrm{a}$ & $2.37 \mathrm{ab}$ & $2.65 \mathrm{a}$ & $2.82 \mathrm{a}$ & $2.93 \mathrm{ab}$ \\
$p$ & 0.0004 & 0.0213 & 0.0042 & 0.0017 & 0.0008 & 0.0001 \\
\hline
\end{tabular}

${ }^{\dagger}=$ diferentes letras dentro de la columna indican diferencias significativas de acuerdo a la prueba de Tukey $(p=0.05)$.

El diámetro de la corona en el estrato alto varió de $2.43 \mathrm{~cm}$ en la semana 20 a $3.09 \mathrm{~cm}$ en la semana 30 , con un crecimiento promedio por semana de $0.06 \mathrm{~cm}$, mayor que lo reportado por Tariq et al. (2013) donde obtuvo diámetros de 1.06 a $1.4 \mathrm{~cm}$ probando diferentes densidades de plantas y sustratos en hidroponía en fresa, en donde el mayor diámetro se obtuvo con una mezcla de sustratos arena, estiércol y limo $(1: 1: 1)$ con una densidad de plantas de 22 plantas $\cdot \mathrm{m}^{-2}$. En el estrato medio alto se observó un incremento en el diámetro de la corona de $0.03 \mathrm{~cm}$ por semana, lo cual correspondió a la mitad del promedio en las plantas del estrato alto, y en el estrato medio bajo se mantuvo el mismo tamaño entre estas 10 semanas (de las 20 a las 30 SDT).

De la misma manera que en la variable número de hojas, la luz pudo influir (Díaz-Pérez, 2013) en el tamaño del diámetro de la corona, ya que, a menor número de hojas, menor grosor de corona en la planta de fresa. La floración comenzó 20 SDT. Sin embargo, Hidaka et al. (2017) reportan que la floración en fresa comienza 6 SDT. En este estudio el retraso en la floración podría ser atribuido a la temperatura alta y un fotoperiodo largo, ya que la baja temperatura y días cortos inducen a la diferenciación floral (Miyoshi et al., 2013).

Dentro del invernadero, la temperatura máxima promedio (TMaxP) durante los meses de marzo a junio fue de $35^{\circ} \mathrm{C}$ y la mínima promedio (TminP) $15^{\circ} \mathrm{C}$, de julio a octubre la TMaxP fue de $32{ }^{\circ} \mathrm{C}$ y la TMinP $14.5^{\circ} \mathrm{C}$. La mínima y máxima registrada a lo largo del ciclo del cultivo fueron $4{ }^{\circ} \mathrm{C}$ a $38^{\circ} \mathrm{C}$, respectivamente. Neri et al. (2012) menciona que el desarrollo de botones florales en etapa temprana se realiza en un rango óptimo de temperatura de $26.7^{\circ} \mathrm{C}$ día/ $15.6^{\circ} \mathrm{C}$ noche, porque las altas temperatura inhiben la floración parcialmente $\left(>26^{\circ} \mathrm{C}\right)$ o totalmente $\left(>30^{\circ} \mathrm{C}\right)$.

Variable calidad y rendimiento de fruto. Estas variables registraron comportamiento similar al descrito en número de hojas y diámetro de corona; es decir, sólo el factor estrato tuvo efecto significativo. Las plantas ubicadas en la parte superior respecto a las plantas del estrato medio alto 
y medio bajo presentaron mayor diámetro de fruto (Cuadro 2). Las plantas del estrato alto produjeron estadísticamente igual número de frutos (Cuadro 3), que las de estrato bajo en las 22 y 24 SDT, con una tendencia de ser mayor en el estrato alto. En el estrato medio bajo el diámetro de los frutos fue menor que el estrato alto de la 22 a la 30 SDT.

Cuadro 2. Diámetro de fruto en dos cultivares de fresa como resultado de cuatro estratos de plantas en bolsas horizontales en sistema hidropónico piramidal.

\begin{tabular}{ccccccc}
\hline \multirow{2}{*}{ Factor estrato } & \multicolumn{5}{c}{ Semanas después del trasplante } \\
\cline { 2 - 7 } & 20 & 22 & 24 & 26 & 28 & 30 \\
\hline Alto & $1.48 \mathrm{a}^{\dagger}$ & $1.88 \mathrm{a}$ & $2.11 \mathrm{a}$ & $1.84 \mathrm{a}$ & $1.79 \mathrm{a}$ & $1.95 \mathrm{a}$ \\
Medio alto & $0.58 \mathrm{a}$ & $0.65 \mathrm{~b}$ & $0.82 \mathrm{~b}$ & $0.94 \mathrm{ab}$ & $1.09 \mathrm{ab}$ & $1.08 \mathrm{ab}$ \\
Medio bajo & $0.62 \mathrm{a}$ & $0.73 \mathrm{~b}$ & $0.51 \mathrm{~b}$ & $0.67 \mathrm{~b}$ & $0.68 \mathrm{~b}$ & $0.69 \mathrm{~b}$ \\
Bajo & $1.07 \mathrm{a}$ & $1.16 \mathrm{ab}$ & $1.41 \mathrm{ab}$ & $1.22 \mathrm{ab}$ & $1.62 \mathrm{ab}$ & $1.47 \mathrm{ab}$ \\
$p$ & 0.0604 & 0.006 & 0.0001 & 0.0283 & 0.0337 & 0.0107 \\
\hline
\end{tabular}

$\dagger=$ diferentes letras dentro de las columnas indican diferencias significativas con la prueba de Tukey $(p=0.05)$.

Cuadro 3. Número de frutos de fresas por planta en dos cultivares de fresa como resultado de cuatro estratos con bolsas horizontales en sistema de producción piramidal.

\begin{tabular}{ccccccc}
\hline \multirow{2}{*}{ Factor estrato } & \multicolumn{6}{c}{ Semanas después del trasplante } \\
\cline { 2 - 6 } & 20 & 22 & 24 & 26 & 28 & 30 \\
\hline Alto & $3.33 \mathrm{a}^{\dagger}$ & $5.88 \mathrm{a}$ & $7 \mathrm{a}$ & $6.66 \mathrm{a}$ & $6.77 \mathrm{a}$ & $6.44 \mathrm{a}$ \\
Medio alto & $1.22 \mathrm{a}$ & $1.33 \mathrm{~b}$ & $2.66 \mathrm{~b}$ & $2.66 \mathrm{~b}$ & $2.88 \mathrm{a}$ & $3.33 \mathrm{a}$ \\
Medio bajo & $1.33 \mathrm{a}$ & $2 \mathrm{~b}$ & $2.22 \mathrm{~b}$ & $1.88 \mathrm{~b}$ & $3.77 \mathrm{a}$ & $4 \mathrm{a}$ \\
Bajo & $2.88 \mathrm{a}$ & $3.55 \mathrm{ab}$ & $4.66 \mathrm{ab}$ & $4.44 \mathrm{ab}$ & $3.88 \mathrm{a}$ & $6.33 \mathrm{a}$ \\
$p$ & 0.0561 & 0.002 & 0.0071 & 0.0042 & 0.1805 & 0.2595 \\
\hline
\end{tabular}

${ }^{\dagger}=$ diferentes letras dentro de la columna indican diferencias significativas con la prueba de Tukey $(p=0.05)$.

El rendimiento fue mayor en el estrato alto de la pirámide, excepto en la semana 30 donde el rendimiento de planta fue similar al estrato bajo (Figura 3A). Las plantas del estrato bajo tuvieron una tendencia ascendente en el rendimiento, mientras tanto la curva del estrato alto tuvo un comportamiento decreciente al final del experimento (Figura 3B). De acuerdo con Ledesma et al. (2008), la temperatura y la duración del día juegan un papel fundamental que afecta la producción de frutos. Ledesma y Kawabata (2016) mencionan que existe una disminución en tamaño y peso de fruto causado por altas temperaturas (mayor a $32{ }^{\circ} \mathrm{C}$ por más de $4 \mathrm{~h}$ ) en los cultivares de fresa, las temperaturas menores a $2{ }^{\circ} \mathrm{C}$ y mayores a $35{ }^{\circ} \mathrm{C}$ por periodos prolongados provocan desvitalización del polen, aborto floral y malformaciones de fruto. 


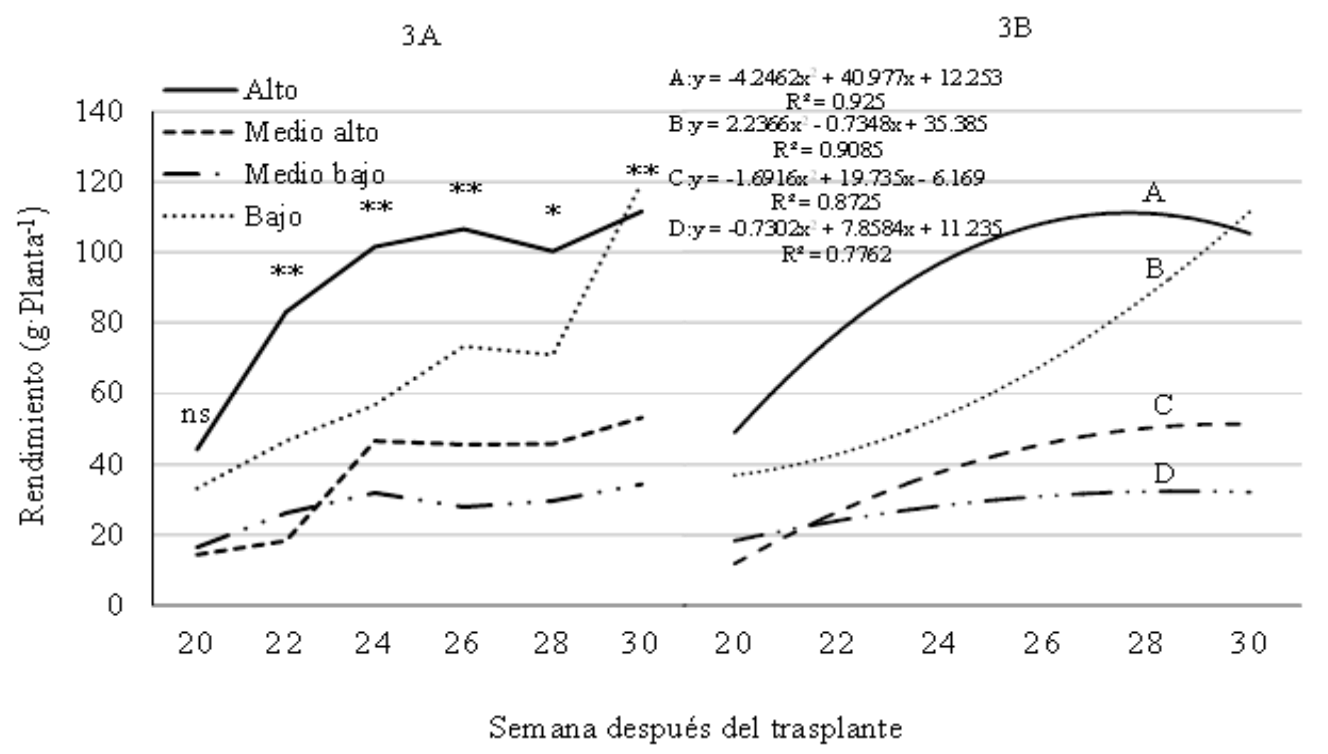

Figura 3. Rendimiento acumulado de frutos fresa de invernadero producida en cuatro diferentes estratos de sistema de producción con estructura piramidal. (3A) las curvas conectan medias; datos evaluados mediante análisis de varianza (General Linear Model, SAS); (3B) curvas calculadas mediante regresión lineal. $\mathrm{ns},{ }^{*},{ }^{* *},{ }^{* * *}=$ no significativo y significativo a $p \leq$ $0.05,0.01$ y 0.001 , respectivamente.

Probablemente los cultivares 'Festival' y 'San Andreas' respondieron favorablemente al estrés por alta temperatura, ya que de julio a octubre se tuvieron temperaturas máximas de $38{ }^{\circ} \mathrm{C}$, pero de una duración menor a las $4 \mathrm{~h}$. Caruso el al. (2011) reportan que la duración del estrés por alta temperatura en fresa afecta el número de frutos. Estos autores mencionan que el periodo en el que se cultiva la planta tiene gran relevancia para la producción de fresa, ya que las horas frío son cruciales para la fresa, concordando con nuestro estudio, ya que probablemente también la acumulación excesiva de horas frío evitó que la planta se desarrollara en mejores condiciones.

Los sólidos solubles totales sólo fueron diferentes en la 24, 26 y 30 SDT en el tratamiento con el estrato alto y bajo donde se tienen los valores más altos (mejor calidad), aunque estadísticamente los valores del estrato medio alto y medio bajo no fueron diferentes del estrato bajo (Cuadro 4).

Cuadro 4. Sólidos solubles totales en los frutos de dos cultivares de fresa ( ${ }^{\circ}$ Brix), producidos en cuatro estratos en bolsas horizontales en sistema hidropónico piramidal.

\begin{tabular}{ccccccc}
\hline \multirow{2}{*}{ Factor estrato } & \multicolumn{6}{c}{ Semana después del trasplante } \\
\cline { 2 - 7 } & 20 & 22 & 24 & 26 & 28 & 30 \\
\hline Alto & $5.6 \mathrm{a}^{\dagger}$ & $7.3 \mathrm{a}$ & $8 \mathrm{a}$ & $7.4 \mathrm{a}$ & $6.7 \mathrm{a}$ & $7.3 \mathrm{a}$ \\
Medio alto & $2.1 \mathrm{a}$ & $6.9 \mathrm{a}$ & $3.1 \mathrm{~b}$ & $2.8 \mathrm{~b}$ & $3.8 \mathrm{~b}$ & $4.5 \mathrm{ab}$ \\
Medio bajo & $2.5 \mathrm{a}$ & $2.8 \mathrm{a}$ & $1.9 \mathrm{~b}$ & $2.8 \mathrm{~b}$ & $2.8 \mathrm{~b}$ & $2.4 \mathrm{~b}$ \\
Bajo & $3.4 \mathrm{a}$ & $4.3 \mathrm{a}$ & $5.2 \mathrm{ab}$ & $4.9 \mathrm{ab}$ & $6.1 \mathrm{a}$ & $6 \mathrm{ab}$ \\
$p$ & 0.06 & 0.5704 & 0.0002 & 0.0061 & 0.0369 & 0.0132 \\
\hline
\end{tabular}

$\dagger=$ diferentes letras dentro de la columna indican diferencias significativas con la prueba de Tukey $(p=0.05)$. 
Los sólidos solubles totales fueron mayores cuando se incrementaron los rendimientos (Figura 4), el rendimiento también aumentó cuando se incrementó la incidencia de la radiación solar. La radiación solar se encuentra asociada con el contenido de sólidos soluble en el fruto. Díaz-Pérez (2014) reportó que la cantidad de sólidos soluble totales decrece cuando se incrementa el nivel de sombra en chile morrón (Capsicum annuum L.). Así, al tener mayor sombreo en los estratos medio alto y medio bajo se tiene una menor cantidad de sólidos solubles en el fruto, estratificado de la misma manera que las demás variables observadas.

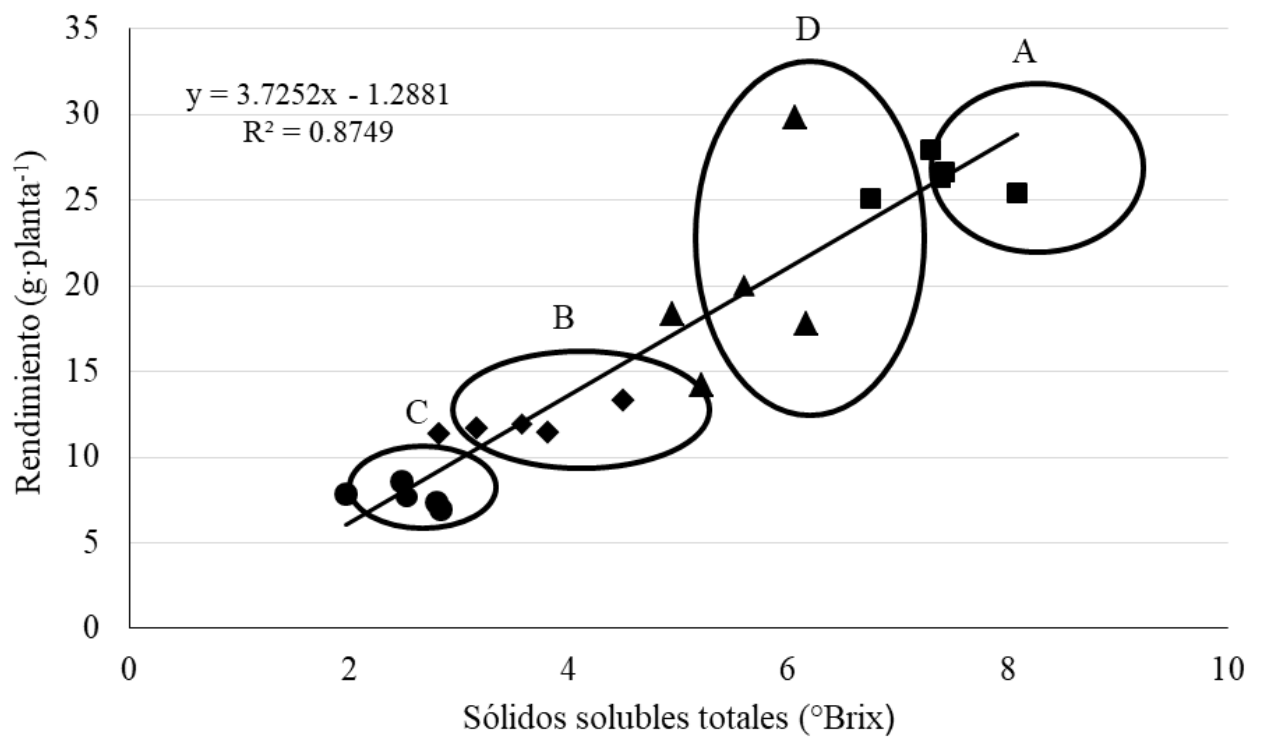

Figura 4. Relación entre sólidos solubles totales y rendimiento como resultado de cuatro estratos con bolsas horizontales en sistema hidropónico piramidal; a través, del análisis de regresión lineal. $\mathrm{A}=$ alto, $\mathrm{B}=$ medio alto, $\mathrm{C}=$ medio bajo, $\mathrm{D}=$ bajo .

Los rendimientos obtenidos por planta concuerdan con la tendencia en las variables: número de fruto y diámetro de fruto, ya que el estrato alto, medio alto y medio bajo, tiene el mismo comportamiento decreciente en rendimiento por planta, ajustándose a la probabilidad de que la sombra actuó sobre el sistema realizando un patrón sobre los tres estratos en los cuales nos muestra que la variabilidad de luz que incidió en el sistema afectó a las plantas en su conjunto, esto concuerda con lo reportado por Wang y Wang (2014) ya que menciona que la intensidad de luz es un factor clave para la mejora de la producción de fresa en un sistema hidropónico.

Los resultados en producción en este estudio fueron bajos en comparación con los presentados por Miranda et al. (2014) en un sistema hidropónico en bolsas y canales de fibra de coco ya que ellos obtuvieron 684 y $1407 \mathrm{~g}$ planta $^{-1}$ manejando una densidad de 10 y 13 plantas $\mathrm{m}^{-2}$, respectivamente. Sin embargo, el rendimiento total fue más alto en este estudio. Ya que usando una densidad de 43 plantas $\mathrm{m}^{-2}$, se obtuvo una producción de $23.5 \mathrm{~kg} \mathrm{~m}^{-2}$ en el estrato alto del sistema piramidal (Cuadro 5), por lo tanto, uno de los beneficios del sistema piramidal es la capacidad de poder incrementar la densidad de plantas por metro cuadrado y la optimización del espacio. 
Cuadro 5. Rendimiento acumulado de fresa por planta y por metro cuadrado en dos cultivares de fresa como resultado de cuatro estratos en sistema hidropónico piramidal.

\begin{tabular}{ccc}
\hline & \multicolumn{2}{c}{ Rendimiento } \\
\cline { 2 - 3 } Estrato & $\left(\mathrm{g} \mathrm{planta}^{-1}\right)$ & $\left(\mathrm{kg} \mathrm{m}^{-2}\right)$ \\
\hline Alto & $547.63 \mathrm{a}^{\dagger}$ & $23.5 \mathrm{a}$ \\
Medio alto & $223.49 \mathrm{~b}$ & $9.6 \mathrm{~b}$ \\
Medio bajo & $165.99 \mathrm{~b}$ & $7.1 \mathrm{~b}$ \\
Bajo & $400.41 \mathrm{ab}$ & $17.2 \mathrm{ab}$ \\
$p$ & 0.0007 & 0.0007 \\
\hline
\end{tabular}

$\dagger=$ diferentes letras dentro de cada columna indican diferencias significativas con la prueba de Tukey $(p=0.05)$.

Los resultados obtenidos en este estudio también fueron más altos que el reportado por Paranjpe $e t$ al. (2003), en este caso por metro cuadrado, ya que reportó rendimiento de 7 a $9 \mathrm{~kg} \mathrm{~m}^{-2}$ en un sistema hidropónico, en un invernadero con control de clima, con producción en los meses de invierno en Florida, EUA.

\section{Conclusiones}

El desarrollo de las plantas de fresa en el sistema piramidal mostró que los cultivares 'Festival' y 'San Andreas'; y las densidades 6, 8 y 10 plantas por bolsa no mostraron diferencias significativas entre tratamientos. El sistema piramidal mostró que en el estrato alto las plantas tuvieron mayor: número de hojas, diámetro de la corona, diámetro de fruto, número de frutos, contenido de sólidos soluble y rendimiento.

\section{Literatura citada}

Avitia-García, E.; Pineda-Pineda, J.; Castillo-González, A. M.; Trejo-Téllez, L. I.; Corona-Torres, T. y Cervantes-Urbán, E. 2014. Extracción nutrimental en fresa (Fragaria x ananassa Duch.). Rev. Mex. Cienc. Agríc. 5(3):519-524.

Benke, K. and Tomkins, B. 2017. Future food-production systems: vertical farming and controlledenvironment agriculture. Sustainability: Science, Practice and Policy. 13(1):13-26. Doi:10.1080/15487733.2017.1394054.

Cantliffe, D. J.; Castellanos, J. Z. and Paranjpe, A. V. 2007. Yield and quality of greenhouse-grown strawberries as affected by nitrogen level in coco coir and pine bark media. Procceding of the Florida State Horticultural Society. 120(1):157-161.

Caruso, G.; Villari, G.; Melchionna, G. and Conti, S. 2011. Effects of cultural cycles and nutrient solutions on plant growth, yield and fruit quality of alpine strawberry (Fragaria vesca L.) grown in hydroponics. Sci. Hortic. 129(3):479-485. Doi: 10.1016/j.scienta.2011.04.020.

Cecatto, A. P.; Calvete, E. O.; Nienow, A. A.; Costa, R. C. D.; Mendonça, H. F. C. and Pazzinato, A. C. 2013. Culture systems in the production and quality of strawberry cultivars. Acta Sci. Agron. 35(4):471-478. Doi: 10.4025/actasciagron.v35i4.16552.

Chandler, C. K.; Legard, D. E.; Dunigan, D. D.; Crocker T. E. and Sims, C. A. 2000. Strawberry Festival strawberry. Hortscience. 35(7):1366-1367. Doi: 10.21273/HORTSCI.35.7.1366. 
Çolak, A. and Alan, F. 2017. Molecular characterization of different currant types. Inter. J. Agric. Fores. Life Sci. 1(1):22-26.

Díaz-Pérez, J. C. 2013. Bell pepper (Capsicum annuum L.) crop as affected by shade level: Microenvironment, plant growth, leaf gas exchange, and leaf mineral nutrient concentration. HortScience. 48(2):175-182. Doi:10.21273/HORTSCI.48.2.175.

Díaz-Pérez, J. C. 2014. Bell pepper (Capsicum annuum L.) crop as affected by shade level: fruit yield, quality, and postharvest attributes, and incidence of Phytophthora blight (caused by Phytophthora capsici L.). HortScience. 49(7):891-900. Doi:10.21273/HORTSCI.49.7.891.

Eurosemillas. 2020. Variedades de semillas. http://www.eurosemillas.com/es/variedades/fresa/ item/27-san-andreas.html.

FAOSTAT. 2020. Produccion mundial de fresa. En línea: http://faostat3.fao.org2.

Hernanz, D.; Recamales, Á. F.; Meléndez-Martínez, A. J.; González-Miret, M. L. and Heredia, F. J. 2008. Multivariate statistical analysis of the color anthocyanin relationships in different soilless-grown strawberry genotypes. J. Agric. Food Chem. 56(8):2735-2741. Doi: 10.1021/jf073389j.

Hewitt, E. J. and Smith, T. A. 1974. Plant mineral nutrition. English Universities Press Ltd, United Kingdom. 298 p.

Hidaka, K.; Dan, K.; Imamura, H. and Takayama, T. 2017. Crown-cooling treatment induces earlier flower bud differentiation of strawberry under high air temperatures. Environm. Control Biol. 55(1):21-27. Doi: 10.2525/ecb.55.21.

INEGI. 2020. Estadísticas geográficas. http://www3.inegi.org.mx/sistemas/mexicocifras/default. aspx?e $=24$.

Juárez-Rosete, C. R.; Rodríguez-Mendoza, M. N.; Sandoval-Villa, M. y Muratalla-Lúa, A. 2007. Comparación de tres sistemas de producción de fresa en invernadero. Terra Latinoam. 25(1):17-23.

Karimi, F.; Arunkumar, B.; Asif, M.; Murthy, B. and Venkatesha, K. 2013. Effect of different soilless culture systems on growth, yield and quality of strawberry cv. strawberry festival. Inter. J. Agric. Sci. 9(1):366-372.

Keutgen, A. J. and Pawelzik, E. 2007. Cultivar-dependent cell wall modification of strawberry fruit under $\mathrm{NaCl}$ salinity stress. J. Agric. Food Chem. 55(18):7580-7585. Doi: 10.1021/jf071216o.

Kratky, B. A. 2005. Growing lettuce in three non-aerated, non-circulated hydroponic systems. J. Vegetable Sci. 11(2):35-41. Doi: 10.1300/J484v11n02_04.

Ledesma, N. A.; Nakata, M. and Sugiyama, N. 2008. Effect of high temperature stress on the reproductive growth of strawberry cvs. 'Nyoho' and 'Toyonoka'. Sci. Hortic. 116(2):186193. Doi: 1016/j.scienta.2007.12.010.

Ledesma, N. and S. Kawabata. 2016. Responses of two strawberry cultivars to severe high temperature stress at different flower development stages. Sci. Hortic. 211(1):319-327. Doi: 10.1016/j.scienta.2016.09.007.

López-Pérez, L.; Cárdenas-Navarro, R.; Lobit, P.; Martínez-Castro, O. y Escalante-Linares, O. 2005. Selección de un sustrato para el crecimiento de fresa en hidroponía. Rev. Fitotec. Mex. 28(2):171-174.

Martínez, F.; Oliveira, J. A.; Calvete, E. O. and Palencia, P. 2017. Influence of growth medium on yield, quality indexes and SPAD values in strawberry plants. Sci. Hortic. 217(1):17-27. Doi: 10.1016/j.scienta.2017.01.024. 
Miranda, F. R. D.; Silva, V. B. D.; Santos, F. S. R. D.; Rossetti A. G. and Silva, C. D. F. B. D. 2014. Production of strawberry cultivars in closed hydroponic systems and coconut fibre substrate. Rev. Cienc. Agron. 45(4):833-841. Doi: 10.1590/S1806-66902014000400022.

Miyoshi, Y.; Hidaka, K.; Okayasu, T.; Hirano, O.; Yasutake, D. and Kitano, M. 2013. Approach to local environment control for stable production of strawberry. Japan. IFAC Proceedings Volumes. 46(4):58-61. Doi: 10.3182/20130327-3-JP-3017.00016.

Neri, D.; Baruzzi, G.; Massetani, F. and Faedi, W. 2012. Strawberry production in forced and protected culture in Europe as a response to climate change. Canada. Canadian J. Plant Sci. 92(6):1021-1036. Doi: 10.4141/cjps2011-276.

Ozeker, E.; Eltez, R. Z.; Tuzel, Y.; Gul, A.; Onal, K. and Tanrysever, A. 1999. Investigation on the effects of different growing media on the yield and quality of strawberries grown in vertical bags. Acta Hortic. 491(1):409-414. Doi: 10.17660/ActaHortic.1999.491.64.

Palencia, P.; Bordonaba, J. G.; Martínez, F. and Terry, L. A. 2016. Investigating the effect of different soilless substrates on strawberry productivity and fruit composition. Sci. Hortic. 203(1):12-19. Doi: 10.1016/j.scienta.2016.03.005.

Paranjpe, A. V.; Cantliffe, D. J.; Lamb, E. M.; Stoffella, P. J. and Powell, C. A. 2003. Winter strawberry production in greenhouses using soilless substrates: an alternative to methyl bromide soil fumigation. United States of America. Procceding of the Florida State Hortic. Soc. 116(1):98-105.

Paranjpe, A.V.; Cantliffe, D. J.; Stoffella, P. J.; Lamb, E. M. and Powell, C. A. 2008. Relationship of plant density to fruit yield of 'Sweet Charlie'strawberry grown in a pine bark soilless medium in a high-roof passively ventilated greenhouse. Sci. Hortic. 115(2):117-123. Doi: 10.1016/j.scienta.2007.08.009.

Recamales, A. F.; Medina, J. L. and Hernanz, D. 2007. Physicochemical characteristics and mineral content of strawberries grown in soil and soilless system. J. Food Quality. 30(5):837-853.

Sánchez del Castillo, F.; González-Molina L.; Moreno-Pérez E. D. C.; Pineda-Pineda J. y Reyes-González, C. E. 2014. Dinámica nutrimental y rendimiento de pepino cultivado en hidroponía con y sin recirculación de la solución nutritiva. Rev. Fitotec. Mex. 37(3):261-269.

Tariq, R.; Qureshi, K. M.; Hassan, I.; Rasheed, M. and Qureshi, U. S. 2013. Effect of planting density and growing media on growth and yield of strawberry. Pakistan J. Agric. Res. 26(2):113-123.

Van Ginkel, S. W.; Igou, T. and Chen, Y. 2017. Energy, water and nutrient impacts of Californiagrown vegetables compared to controlled environmental agriculture systems in Atlanta, GA. United States of America. Resources Conservation and Recycling. 122(1):319-325. Doi: 10.1016/j.resconrec. 03.003B.

Wang, J. and Wang, H. 2014. Effects of shade on strawberries in hydroponic cultivation. China. Acta Hortic. 1049(2):733-736. Doi: 10.17660/ActaHortic.2014.1049.114. 